\title{
Interactions between teaching assistants and students boost engagement in physics labs
}

\author{
Jared B. Stang" \\ Department of Physics and Astronomy, University of British Columbia, \\ 6224 Agricultural Road, Vancouver, British Columbia, V6T 1Z1 Canada \\ Ido Roll ${ }^{\dagger}$ \\ Centre for Teaching, Learning, and Technology, University of British Columbia, \\ 214-1961 East Mall, Vancouver, British Columbia, V6T 1 Z1 Canada
}

(Received 28 June 2013; published 5 September 2014)

\begin{abstract}
Through in-class observations of teaching assistants (TAs) and students in the lab sections of a large introductory physics course, we study which TA behaviors can be used to predict student engagement and, in turn, how this engagement relates to learning. For the TAs, we record data to determine how they adhere to and deliver the lesson plan and how they interact with students during the lab. For the students, we use observations to record the level of student engagement and pretests and post-tests of lab skills to measure learning. We find that the frequency of TA-student interactions, especially those initiated by the TAs, is a positive and significant predictor of student engagement. Interestingly, the length of interactions is not significantly correlated with student engagement. In addition, we find that student engagement was a better predictor of post-test performance than pretest scores. These results shed light on the manner in which students learn how to conduct inquiry and suggest that, by proactively engaging students, TAs may have a positive effect on student engagement, and therefore learning, in the lab.
\end{abstract}

DOI: $10.1103 /$ PhysRevSTPER.10.020117

PACS numbers: 01.40.J-, 01.40.gb

\section{INTRODUCTION}

\section{A. Background}

In science courses at the university level, teaching assistants (TAs) take on a variety of roles and are integral to the successful delivery of many courses [1]. These roles range from marking assignments and proctoring exams to facilitating laboratories and leading tutorials. In large-scale courses, as are typical for first-year undergraduates at large universities, TAs are especially important for learning [1], as in many cases they serve as the sole instructors in the smaller lab or tutorial sections.

Given the large amount of responsibility TAs have in these courses, the question becomes, What effect to the TAs have on student learning? In this study, we focus on two questions of interest: How do TA behaviors in the lab affect student engagement and, in turn, what is the relationship between this engagement and learning in the physics lab? (See Fig. 1.) As explained further below, our focus on student engagement stems from the fact that engagement

\footnotetext{
*jstang@phas.ubc.ca

ido.roll@ubc.ca
}

Published by the American Physical Society under the terms of the Creative Commons Attribution 3.0 License. Further distribution of this work must maintain attribution to the author(s) and the published article's title, journal citation, and DOI. has been found to be a significant predictor of learning $[2,3]$. Studying these relationships will contribute to an understanding of the wider question of how TAs affect students and student learning in the classroom and may provide evidence for the effectiveness of particular instructional strategies.

Clearly, there are many other factors, not included in the model of Fig. 1, that influence student engagement and learning. For example, it may be that a student is very motivated in a particular course due to a personal interest in the material and not because of the actions of the TAs. Also, TA behaviors may affect learning directly, not through engagement. For example, an enthused or clear TA may be more effective. Despite these limitations, this model

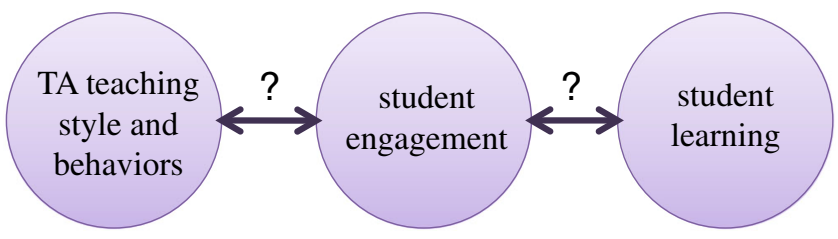

FIG. 1. A simplified model of the TA-student relationship. Each bubble is hypothesized to influence the bubbles immediately next to it. In this paper, we evaluate the correlational links between "TA teaching style and behaviors" and "student engagement" and between student engagement and "student learning." The results are summarized in Fig. 3. 
conveys a simplified representation of the relationship between TAs, students, and learning that is the focus of our current investigation.

We undertake this study in the lab sections of a transformed large-scale first-year algebra based physics course. We begin by reviewing existing literature on TA behaviors and student engagement and providing further motivation for this work. We then describe the context for our investigation and outline the experimental design. Following the results, we discuss the implications for TA training in first-year large enrollment labs.

\section{B. Literature review and motivation}

Research has clearly shown that variations in TAs' beliefs and attitudes influence their behavior as an instructor [4-11]. Subsequently, recent studies have examined the behaviors of TAs, reporting for example on who initiates TA-student interactions [12] and the variations in the types, frequencies, and lengths of TA-student interactions that occur [13-15]. Despite this focus on the actions of TAs and instructors, the literature has not yet made connections between these behaviors and the student learning process and outcomes. In this study, we seek to understand the potentially important effect of these different TA modalities (such as interaction lengths and whether or not the TAs initiate interactions) on students in the physics lab, thereby addressing this open question.

Student engagement in college classrooms may be conceptualized as "the time and effort students devote to activities that are empirically linked to desired outcomes of college"[16]. Engagement of this general type has been shown to be positively linked to grades and persistence in undergraduate students [2]. The above definition of engagement includes many dimensions [17] and applies across the spectrum of undergraduate experiences. To define engagement in the context of the physics lab, we focus on the dimension of being on task. Thus, we define an engaged student as one that is visibly focused on and occupied with the lab activity. This type of engagement has been shown to positively correlate with learning and grades in the lecture $[18,19]$, in the use of intelligent tutoring software [20], and in general [3]. Student engagement, as defined here, is a variable which only exists during class time. As our unit of observation for both TA behaviors and student engagement is then the single lab period (see Sec. II), this allows us to evaluate the possible impact of TAs on students at the same level. The importance of engagement for learning and the practical consideration of evaluating a student outcome in the single lab period motivate our focus on student engagement.

The second question in our study investigates the relationship between engagement, as defined above, and learning. The lab in which this study is undertaken is a hands-on inquiry-based lab. Then, in order to learn, students are expected to engage with the lab content, as there are no other opportunities for them to participate in the learning activities (for example, through lecture notes at home). We therefore hypothesize that in this context engagement will be correlated with learning. Results connecting engagement with learning in this specific situation will help to understand the effect of TA behaviors on student learning and contribute to the existing literature, reviewed above, on the general positive relationship between student engagement and learning.

\section{METHOD}

\section{A. Design}

This study took place in the lab sections of a large-scale first-year physics course. Within the inquiry-based lab, in which the TAs are the sole instructors, we look, in particular, at two aspects of TA teaching style and behaviors: how the TAs adhere to and deliver the lesson plan [21] and how the TAs interact with students during the work session. The specific TA behaviors we record (in italics) are as follows:

- How the TAs adhere to and deliver the lesson plan.

(1) Behaviors outside of the standard TA script.

- How the TAs interact with students during the work session.

(2) Number of interactions.

(3) Who initiates the interactions.

(4) Length of interactions.

Our main strategy for data collection was to perform observations of the lab. From an unobtrusive vantage point at the back of the lab room, observers were able to record data on the TA behaviors listed above.

This study further evaluates two student factors: engagement and learning. We define an engaged student as one that visibly has their attention focused on the lab activity. As behavioral measures are often more reliable than self reports [22,23], we measure engagement through observations. By periodically circulating the room, observers measured snapshots of student engagement in the lab. As discussed in more detail in Sec. II C 1, strategies to minimize the disturbance of the observations on the classroom were utilized. The observations were completed during one lab session in a typical week in the middle of the term. Finally, learning was measured by giving students a lab exam on the first and last weeks of the term.

\section{B. Participants and description of lab}

We undertake this study in Physics 100, a first-year introductory physics course at the University of British Columbia (UBC), a large research-intensive university. 713 
students were spread between 17 lab sections. The students in this course are primarily in the life sciences and, for most of them, this will be their only physics course. In addition, the majority of the students are first-year, first-term university students.

The lab portion of Physics 100, which consists of weekly sessions, each $80 \mathrm{~min}$ in length, has been extensively revamped in recent years to utilize an inquirybased approach. The learning goals for the lab are aimed at the development of a general set of scientific and data analysis tools rather than being focused on content knowledge. A typical lab begins with an introduction and a set of clicker questions, followed by an extended period of students working in pairs, before ending with a summary discussion and another set of clicker questions. The average number of students in each lab was 39 while the largest and smallest sections had 46 and 25 students in them.

During the observation week, the main task of the lab was to collect and extrapolate a data set in order to make a prediction for a future experiment. By measuring the time it took a coffee filter to fall to the ground from heights below $1 \mathrm{~m}$, students had to predict the time it would take for the coffee filter to fall from a height of $2 \mathrm{~m}$. To complete this inquiry-based activity, students were required to design and carry out an experiment that would allow them to predict the falling time from the desired height, for example, by performing a linear fit to data for various heights below $1 \mathrm{~m}$. Once each pair of students had made a prediction, one TA would perform (or get students to perform) the experiment at the height of $2 \mathrm{~m}$, while the other would collect the predictions from each group. We define the "student working period" in the lab as the time during which students are working to make their predictions, beginning after the introduction and ending once the TAs begin performing the experiment at a height of $2 \mathrm{~m}$ or soliciting student predictions. The lab ended with a discussion comparing the predictions to the measured results.

In Physics 100, the TAs are the instructors in the lab, having full control of the section. We exclude one of the lab sections from our analysis, because in the observation week there was a replacement TA, as lab norms that may have been established with the regular TA may have been different than the results of our observations. A group of 10 TAs, consisting of 9 males and 1 female, facilitated the 16 remaining lab sections in pairs. Each pair of TAs were assigned to 2 consecutive lab sections, and each TA was assigned to 2 or 4 sections. A TA assigned to 4 sections then taught two different instances of consecutive labs, with a different TA partner for each instance. The TAs attended weekly meetings in which the upcoming lab was reviewed.
Six of the 10 TAs were first-year graduate students at UBC and had no prior teaching experience, while 2 TAs had more than one year of TA experience at UBC. The TAs ranged in age from 22 to 28 , with a median age of 24 . Upon beginning graduate school at UBC, each of the firstyear TAs underwent an $8 \mathrm{~h}$ TA professional development workshop in addition to a $3 \mathrm{~h}$ Physics 100 specific workshop [24].

\section{Data collection and analysis}

\section{Data collection}

To observe the TAs, we developed the TA observation form (Appendix B) to record a timeline of the TAs' activities during the lab. On the form, we identified three main TA behaviors.

(1) "Talking to class": The TA is addressing the entire class (for example, leading a classroom discussion).

(2) "Inactive": The TA is not available to students (for example, the TA may be out of the room or talking to the other TA).

(3) "Active": The TA is either helping students or is available to help students.

Under the active category, the number, length, and initiator of interactions with students was recorded. In addition, our form allowed us to record other section data, including the progression of the lab (through the introduction, clicker questions, etc.). A category titled "extras" was used to record any TA behavior that was outside of the main script of the lab. (See Appendix C for a list of extra behaviors recorded.) A completed TA observation form allows us to see what the TA was doing at each moment during the observed lab.

To measure student engagement, we adapted the BakerRodrigo observation method protocol [25,26], developing the on-off task form (Appendix D). This form consisted of a spatial map of the lab with empty squares to represent student positions at each lab bench. To fill out the form, the observer looked at the lab bench. If a student was on task, as judged by the observer at a glance, a check mark was placed in the corresponding square on the form, while if a student was off task, an " $x$ " was placed in the square. Some clues observers used to assign the binary engagement value to each student include whether or not the student was involved in an off task behavior and whether students were attending to their partner and their task through eye contact, body position, and verbal cues. For example, a student that appeared to be writing on their lab worksheet was judged as on task and a student that was looking at their cell phone was marked as off task. The on-off task form was completed at intervals of $10 \mathrm{~min}$ during the lab, giving snapshots of the engagement level of the class. The fractional engagement for a snapshot is defined as the 
number of on task students (number of check marks) divided by the total number of students present in the section. Both the TA observation form and the on-off task form underwent iterative design.

Four observers observed 7-9 sections each. Two observers observed each lab section, with each observer documenting the actions of one TA. A set of practice observations were undertaken prior to the observation week and used to confirm interrater reliability. During this 15 min practice observation, all four observers recorded $90 \%-93 \%$ agreement on the number of interactions (12.5 \pm 1.3 recorded interactions) and the number of short interactions (less than $1 \mathrm{~min}$ long) $(11.0 \pm 0.8)$. Far fewer long interactions $(1.5 \pm 0.6)$ and extra off-script TA behaviors $(2.0 \pm 0.8)$ were recorded and as such these categories did not show as good of an agreement. One snapshot of the student engagement level of the class was taken, giving a fractional student engagement of $0.87 \pm 0.06$ between the observers, showing agreement of $93 \%$. All disagreements between the observers were on two benches which appeared off task by two of the observers but on task by two other observers. Therefore, there is agreement within the observers on which benches were off task and it appears that most of the variability in fractional engagement is due to the differences in the precise time at which the students were observed. We expect that such effects will be mitigated by recording multiple snapshots.

To record data on the TA observation form, the observers watched the TA from an unobtrusive vantage point at the back of the classroom. In order to not interfere with the lab, observers made a conscious effort to not talk or interact with the TAs or students in the lab. Further, the TAs were told only that the observers would be observing the classroom. They were not told that the observers would be recording specific data about the TA, nor were they told that the observers were not. We note also that these labs were often observed by visitors who were unfamiliar to most TAs and students. These design choices were made so that the effect of observers is not different from other observers who frequently attend the lab, and thus should not affect the natural behaviors of students or TAs. Because of the observation style, any data about the style, content, and quality of the TA-student interactions is outside the scope of this study. To complete the on-off task form required the observer to move from the back of the lab room, in order to properly observe the engagement of the students. Typically, the observer would take a 1-2 min to walk around the classroom and fill out the form, again consciously avoiding interactions with students and TAs. To avoid an effect in which students in the lab might be motivated to appear to be working if they feel like they are being observed, the observers made an effort to observe the students discreetly. One strategy in this regard, previously used by Baker et al. [25,27], was to stand near one lab bench while observing a different bench.

To evaluate the relationship between engagement, interactions, and learning, we evaluated student learning using prelab and postlab exams. The lab exams were given during the first and last week of the lab. The exams were adapted from the Concise Data Processing Assessment [28] and Lawson's Classroom Test of Scientific Reasoning [29]. The tests were given without prior notice. All items were multiple choice, as shown in Appendix E.

\section{Analysis}

We compiled the TA data to give results on a per-lab basis, as student engagement is expected to be a product of the behaviors of both TAs in a section. For the number of TA-student interactions and the student engagement measurements, we use data from the student working period only. This is the only period during the lab in which these measurements are defined and relevant. In addition, from the perspective of the TAs, this student working period is rather free form. Within the main goal of supporting the students' activities, differences in TA facilitation style are expected to manifest themselves as different TA behaviors during this time. Since the time each lab section spends in this student working period varies, in order to compare across sections the number of TA-student interactions is normalized by the length of the section's student working period, giving a frequency of interactions. The calculation of this frequency takes into account moments in which observers were not observing due to a variety of reasons. (Usually, this was due to observers completing the on-off task form.)

The length of interactions and the frequency of interactions are interdependent, as the longer the interactions are, the fewer interactions one has time to do. In order to disentangle the effect of the length of interactions from the number of interactions, we look at the ratio of short to long interactions, which gives a unitless measure that does not depend on the frequency of interactions. Through the 16 sections, 564 TA-student interactions were recorded. Of these, 391 were less than a minute. Therefore, we have taken interactions lasting greater than or equal to a minute to be "long," with interactions lasting less than a minute being "short."

We recorded the number of behaviors outside of the standard TA script through the entire lab.

In these labs, it is customary to move on only when all students have completed the task, so that there is typically some down time for quick students. It is important that we measure engagement during the time in which students choose to be on task, before students have finished their lab activity. We restrict the engagement results to the relevant 
part of the student working period by taking only the first three student engagement snapshots recorded. These three snapshots span a time of $19.6 \pm 3.3$ minutes.

Finally, we have the student learning data. As we combine TA data by section, we average the lab test performance across all students in each section. Overall, students improved significantly on test items that were shared by the pretests and post-tests. Pretest: $66 \% \pm 5 \%$; post-test: $76 \% \pm 4 \% . t(15)=10.1, p<0.0001$.

\section{Limitations}

One of the main limitations of this study is that the observational data are restricted to a single lab. This is especially challenging for examining the link between engagement and learning, as we correlate measures from a single snapshot (student engagement) with outcomes of the overall lab course (student learning). Thus, our study does not take into account the week-to-week variations that occur in many important factors, such as TA behaviors, lab style, student attitudes, etc. In the context of this study, these variations would likely introduce random noise and weaken possible relationships. Finding a significant correlation between engagement and learning in this highly noisy environment, as we do below (Sec. III B 2), thus provides evidence that such a relationship does exist. It would be very interesting to study classroom engagement over the course of a semester to provide more information about the possible connection between our single lab observation and semester-long trends. Such study would help to confirm or refute the results presented here.

Another important limitation is our inability to access more fine-grained information about the style and content (and quality) of TA-student interactions. It would be expected that the quality of interactions plays a large role in determining the student response to the TAs. In addition, evaluating the content of the interactions would provide important data about which types of interactions are useful for student learning. For example, does a TA who is sympathetic to students' thinking provide better support

TABLE I. The time spent in each stage of the lab for all 16 observed sections. The student working period, during which students were working in pairs to complete the activity, was the longest stage of the lab.

\begin{tabular}{lcc}
\hline \hline Lab stage & Mean $(\mathrm{m})$ & $\sigma(\mathrm{m})$ \\
\hline Discussion of previous homework & 7.9 & 3.1 \\
Introduction and first clicker questions & 11.3 & 2.7 \\
$\quad \begin{array}{l}\text { Student working period: Students working } \\
\quad \text { to make predictions }\end{array}$ & 32.0 & 6.3 \\
TAs perform experiment, class discussion, & 26.3 & 6.6 \\
$\quad$ and final clicker questions & & \\
\hline \hline
\end{tabular}

TABLE II. TA behavior data descriptives.

\begin{tabular}{llllr}
\hline \hline TA behavior & Mean & Max & Min & $\sigma$ \\
\hline $\begin{array}{l}\text { No. of behaviors outside of the } \\
\quad 9.4\end{array}$ & 22 & 4 & 4.6 \\
$\quad \begin{array}{l}\text { standard TA script } \\
\begin{array}{l}\text { Fraction of interactions per minute } \\
\quad \text { by the TA }\end{array}\end{array}$ & 1.31 & 2.33 & 0.51 & 0.42 \\
$\begin{array}{l}\text { Ratio of short (<1 min) to } \\
\quad \text { long ( } \geq 1 \text { min) interactions }\end{array}$ & 2.8 & 5.25 & 0.46 & 1.3 \\
\hline \hline
\end{tabular}

for learning [30]? To collect these data would either require the observers to be more intrusive, which would complicate the analysis, or necessitate the use of more advanced technology (i.e., video and audio recording), both of which are outside the scope of our study.

A final concern in our design is the possible presence of an observer effect in the student engagement data, as students may tend to make sure they appear to be working if they feel that someone is watching them. The average engagement level through the three engagement snapshots used in our results is very high, at $88 \%$, and the range is from $67 \%$ to $99 \%$. Any observer effect would push engagement numbers higher, tending to minimize the differences in the data and washing out the effect. Thus, we expect that our method of collecting engagement data is sufficient and that any correlation between the number of TA-student interactions and engagement will be robust.

\section{RESULTS}

\section{A. Descriptives of TA teaching style and engagement results}

We observed a high amount of variability between the 16 lab sections, as summarized in Tables I and II. This wide variation in TA style is evident in the recorded number of behaviors outside the standard TA script $(9.4 \pm 4.6)$. The number of interactions per minute across the sections was

TABLE III. Correlation of TA behaviors with student engagement. At an $\alpha$ level of 0.05 , the frequency of interactions and the frequency of TA-initiated interactions give the only statistically significant correlations.

\begin{tabular}{lcc}
\hline \hline TA behavior & $r(14)$ & $p$ \\
\hline $\begin{array}{l}\text { No. of behaviors outside of the } \\
\quad \text { standard TA script }\end{array}$ & -0.11 & 0.66 \\
No. of interactions per minute & 0.52 & 0.03 \\
- Frequency of TA-initiated interactions & 0.49 & 0.04 \\
- Frequency of student-initiated interactions & 0.052 & 0.84 \\
Ratio of short (<1 min) to & -0.10 & 0.70 \\
$\quad$ long ( $\geq 1$ min) interactions & & \\
\hline \hline
\end{tabular}


$1.31 \pm 0.42 .365$ (or $65 \%$ ) of the 564 interactions were initiated by the TAs [31], while the rest (except for 23 interactions with no marked initiator) were initiated by students. Last, the ratio of short to long interactions was $2.8 \pm 1.3$; the majority of interactions were less than 1 min long.

The student engagement results show a very high fractional engagement, at $0.88 \pm 0.08$, through the first three engagement snapshots.

\section{B. Correlations \\ 1. Correlation of TA behaviors with student engagement}

We correlated student engagement with each of the TA behavior variables, using an $\alpha$ level of 0.05 . These results are shown in Table III. As shown in Fig. 2, the frequency of interactions between TAs and students was a positive and significant predictor of student engagement: $r(14)=0.52$, $p=0.03 . r^{2}=0.27$, suggesting the $27 \%$ of the variability in student engagement per section is explained by the frequency of their interactions with TAs. In addition, the frequency of interactions initiated by TAs is significantly correlated with engagement, $r(14)=0.49, p=0.04$. The frequency of interactions that were initiated by students is not correlated with engagement: $r(14)=0.052$. The other variables were not significantly correlated with student engagement.

\section{Correlation of student engagement with learning}

To evaluate the relationship between student engagement and learning, we calculated the partial correlation with the post-test, controlling for performance on the pretest. The measure of engagement is significantly correlated with learning: partial $r(13)=0.56, p=0.03, r^{2}=0.31$. Thus,

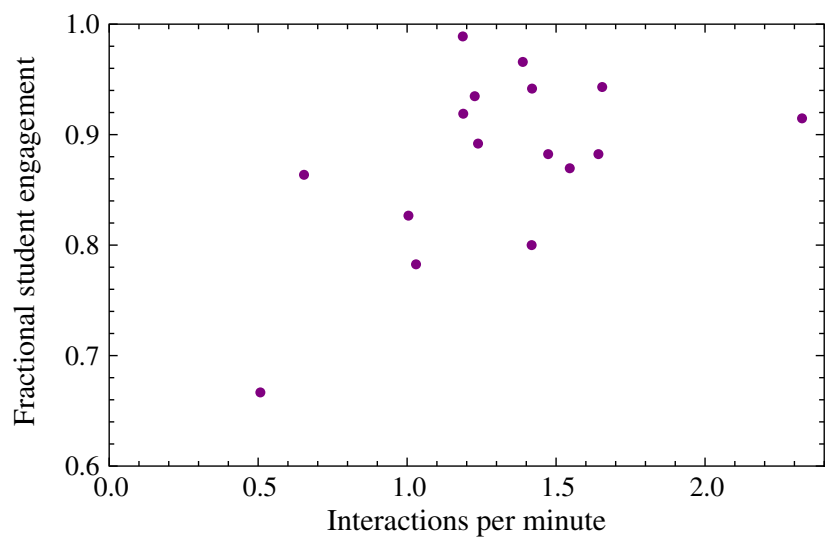

FIG. 2. Fractional student engagement versus number of TA-student interactions per minute during the student working period in the lab. The frequency of interactions is positively correlated with engagement: $r(14)=0.52$.
$31 \%$ of students performance in the lab can be explained by their engagement (as measured in a single session). Performance on the pretest by itself is not significantly correlated with post-test scores: $r(14)=0.32, p=0.23$.

\section{DISCUSSION}

\section{A. Discussion of results}

Of the TA behaviors described in Sect. II and monitored in our study, only the number of interactions per minute has a significant correlation with student engagement. Interestingly, the correlation between interactions and engagement is explained solely by TA-initiated interactions. The rate of student-initiated interactions had no relationship with engagement, while a high rate of TAinitiated interactions is strongly associated with increased engagement. Finally, we found a significant correlation between engagement and learning in this inquiry-based lab. Our results are summarized in Fig. 3.

While our observations are correlational and not causal, suggesting potential explanations for these correlations is of interest. Interactions may positively contribute to motivation and engagement by satisfying students' needs and enabling their success. Also, interactions may reduce disengagement due to a policing effect. The fact that interactions were related to engagement only when initiated by TAs suggests that the relationship is not due to student factors such as intrinsic motivations. Instead, it seems that TA behaviors are the ones that increase engagement, whether perceived as a carrot (due to the given assistance) or a stick (due to their policing effect).

The dependency of the results presented above on the inquiry nature of the lab is of interest. We hypothesize that the correlation between interactions and engagement is not specific to an inquiry-based approach. Our two main inferences, that interactions allow students' needs to be met and that the policing effect of interactions reduces

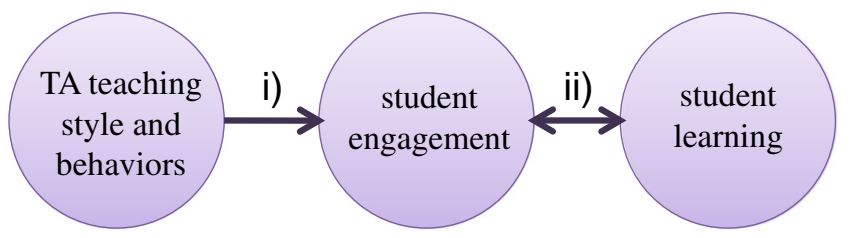

FIG. 3. The main results of our study. We found significant positive correlations (denoted by a solid line) between (i) the frequency of TA-student interactions (a product of TA teaching style and behaviors) and student engagement $[r(14)=0.52]$, (ii) student engagement and student learning [partial $r(13)=0.56$, controlling for pretest]. The correlation between the frequency of TA-student interactions and engagement is explained solely by TA-initiated interactions, implying that it is the TAs that are driving student engagement (indicated by a unidirectional arrow). 
disengagement, are expected to also be valid in a traditional style lab. Compared to an inquiry-based lab, the needs of the students would likely be different in a traditional lab. However, interactions would still allow the TAs to support the students as they work through the lab. Therefore, we speculate that the correlation between interactions and engagement may be generalizable across settings. In contrast, the correlation between engagement and learning may be specific to the inquiry-based lab. In such a lab, students are responsible for generating their own knowledge. If designed properly, there is no avenue for students to learn in the lab without thinking about the process and reflecting on the results, as may be possible in a traditional lab.

It is interesting that the length of interactions does not correlate with the engagement of the students. Although it might be expected that longer interactions would be more positive, as they may be able to better satisfy the students' needs and help the students to feel invested in, our results provide no evidence to support this claim. This suggests that a brief stop by the TA is as effective as an in-depth interaction in keeping students engaged in the lab. A possible explanation is that by initiating a short interaction, the TA opens the door for deep questions from the students, in a sort of "ventilation effect." In this scenario the length of the interaction is not an outcome of TA style, but rather, an adaptive behavior based on students' needs. Then, the length should not affect engagement, as indeed suggested by the data. However, further study is necessary to discern the true effect, if any, of the length of interactions.

The number of off-script TA events does not correlate with student engagement in the lab, even though one might expect that this sort of adaptive instruction might help students to have the tools they need to work through the lab [32]. It could be that off-script behaviors might not be personalized enough to motivate the general student. However, it is more likely that our category was too coarse grained and that only certain types of adaptive instruction have an effect on student engagement. A more detailed study, with the ability to distinguish different types of offscript behaviors, is necessary to establish the specific adaptive behaviors that benefit students.

In addition to the hypotheses tested above, our observations show interesting general results about how TAs spend their time in the class. We observed a large variability in the number of interaction and the number and type of off-script behaviors. In addition to these, there was a substantial variation in the progression of each lab section. It is clear that there are meaningful differences in the facilitation style of different TAs and that these differences are an important factor in determining student engagement and learning.

\section{B. Conclusions and implications}

The correlation of the frequency of TA-initiated interactions with student engagement is an important first step in studying the effect of TAs on student outcomes in a lab course. The results presented here establish a direct relationship between TA behaviors (initiating interactions) and the student response (engagement), thereby demonstrating that variations in TA facilitation styles do have an effect on students and identifying a particular example of this effect. Furthermore, the positive correlation between student engagement and learning in this lab shows that this effect is (indirectly) related to student learning.

In addition to better understanding the effect of TA teaching style on student engagement and learning, results from this study inform and support TA professional development efforts in order to encourage more productive tutoring styles. The positive correlation between TAinitiated interactions and student engagement (and, in turn, the positive correlation between student engagement and learning) suggests that TAs should proactively engage with many students. Since the length of interactions was not found to affect the engagement of students in this course, it is possible that just saying "Hello, is everything okay?" may be as useful as a lengthy discussion in keeping students on task. In our opinion, a more likely explanation is that such short interactions are useful in keeping an eye on student needs and enabling a ventilation effect, giving students an opportunity to access the TAs and opening the door for student questions that turn these brief exchanges into long and meaningful interactions.

\section{ACKNOWLEDGMENTS}

We gratefully acknowledge Doug Bonn, Georg Rieger, and Carl Wieman for useful discussions and Natasha Holmes and Firas Moosvi for comments on the manuscript. This work was funded by the Carl Wieman Science Education Initiative. 


\section{APPENDIX A: TA COPY OF LAB}

In this section, we provide the first three pages of the TA version of the lab worksheet in the observation week (Figs. 4-6). The student version excludes the TA guidelines column on the right of the page.

\section{PHYS 100 EXPERIMENT 7 (week 8) Predictions}

Name: Student \#: Section: Date:

Overview: TA Guidelines

Going over HW (5 min)

- Introduction (10 $\mathrm{min})$

- Data collection (25 min)

Make predictions (20 min)

Test predictions $(10 \mathrm{~min})$

- Summary $(10 \mathrm{~min})$

\section{Introduction (10 min, entire class)}

So far our conclusions were applicable only to situations we tested. For example, we know our own walking speed for the terrains we checked, but cannot predict our walking speed on a new terrain. Today we will try to extend our conclusions to data that was not collected yet. To do that, we will learn to make predictions outside the range of the available data.

Can you think of situations in which collecting data is difficult and predictions are required?

Present the question to students. Examples: - Testing small models before testing the real thing (e.g., planes)

- Testing in normal conditions before extremes (temperature, velocity, etc).

- Predicting events in the future or the past (e.g. the big bang).

\section{Topic: Making predictions.}

clicker 1: Look at the two graphs that you made for homework. What can you say about the temperatures between 1961-2010, compared with the temperatures between 1881-1930?
A. They increased more rapidly
B. The increased at the same rate
C. They increased at a slower rate
D. They did not increase
E. Can't judge without error bars.

The following graphs show the same data that was given to you for your HW.

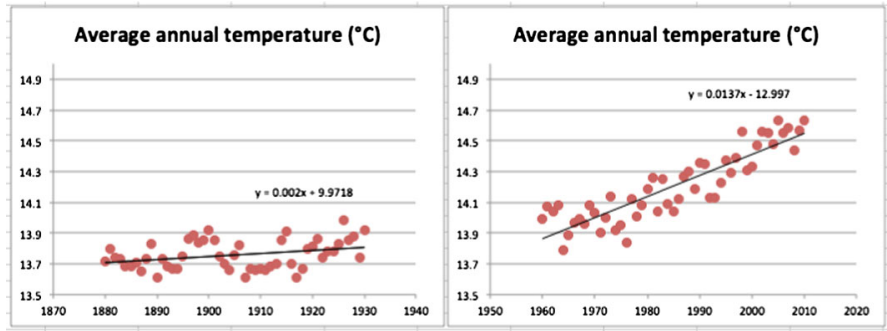

The answer is $A$, they increased more rapidly.

Show that:

a. the temperatures in both periods increased (the line has a positive slope).

b. The temperatures now increase more rapidly (the slope is steeper).

If $>80 \%$ got the clicker right, don't waste time on this and move on.

FIG. 4. Page 1 of the TA version of the lab worksheet. 
Clicker 2: Based on data from the last 50 years, what will be the temperatures in 2050 ?

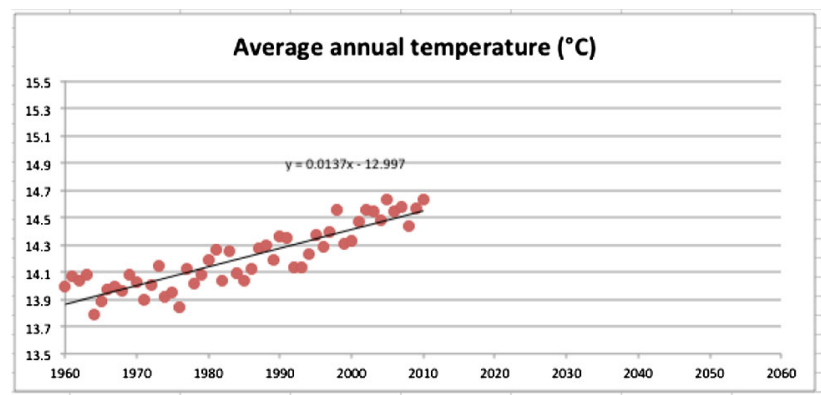

A.13.9-14.7

B.14.7-15.1

C.15.1-16.5

D. 15.5-15.9

E. $15.9-16.3$

The purpose of this

clicker is to show

students that prediction

is hard, and to motivate

the lab.

Give students 2 minutes to work on this.

Show how you use the trendline to make a prediction. Do it in 2 minutes. They will do it later for data they collect.

\section{Problem set up}

As most of you may have heard, on Oct 14, 2012, Felix Baumgartner jumped from a height of 39 kilometers ( $24 \mathrm{mi}$ ), reaching an estimated speed of 1,342 kilometers per hour. He free-fell for 4 minutes and 19 seconds, and then opened his parachute and gilded down to earth.
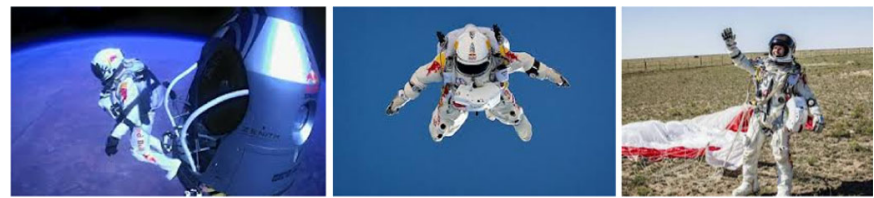

It is obvious that his calculations and testing needed to be accurate....

Since testing parachutes under realistic conditions was impractical, Baumgartner and his team had to test the parachute from lower heights, and make predictions for the full height.

You are in charge of the initial testing. The initial testing will use a model: instead of the actual parachute, you will test a stack of two coffee filters (see image). Your task is to predict how long it will take the coffee filters to fall from 2 meters. However, you can only test them at 1 meter or below.

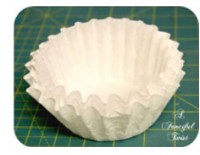

P100 Expt.7, Fall 2012, University of British Columbia

$-2-$

FIG. 5. Page 2 of the TA version of the lab worksheet. 


\section{Tasks}

Task 1: Data collection (25 min, groups of 2)

On the bench you can find coffee filters. Stack two coffee filters. This is your model-parachute.

Your research question is the following:

How long will it take the model parachute to fall from 2 meters?

Collect data that will help you determine that. You can only collect data from the height of 1 meter (bench height) or below.

Drop the filters facing up, as in the image above, and not facing down (that is, they should look like a bowl, not a hat). When facing up, their velocity is more consistent and measurements are more accurate.

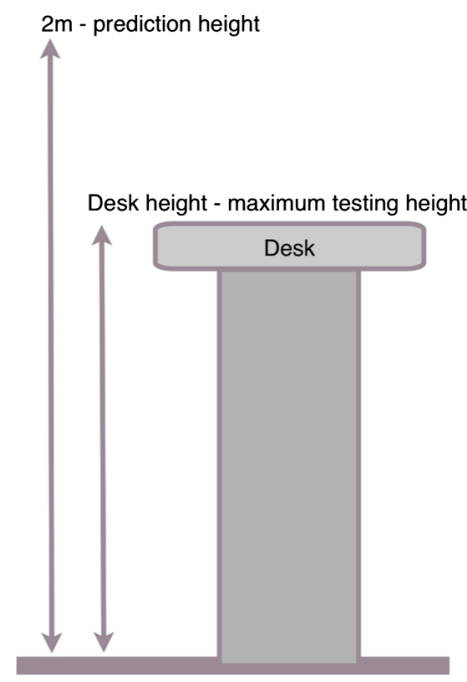

You need to collect enough data to make predictions:

- How many heights will you collect data from? Which heights will you choose?

- How many measurements will you take in each height?

A hint: the answer is not straightforward. Twice the fall from $1 \mathrm{~m}$ will not give you the fall from $2 \mathrm{~m}$.

Document your measurements on the other side of this page.

P100 Expt.7, Fall 2012, University of British Columbia $-3-$
Groups of 6 can exchange ideas, but predictions should be made in groups of 2 , so insure that everyone is working.

Encourage at least 3-4 per height

FIG. 6. Page 3 of the TA version of the lab worksheet. 


\section{APPENDIX B: TA OBSERVATION FORM}

In this Appendix, in Fig. 7, we provide a copy of the TA observation form, used to record TA behaviors.

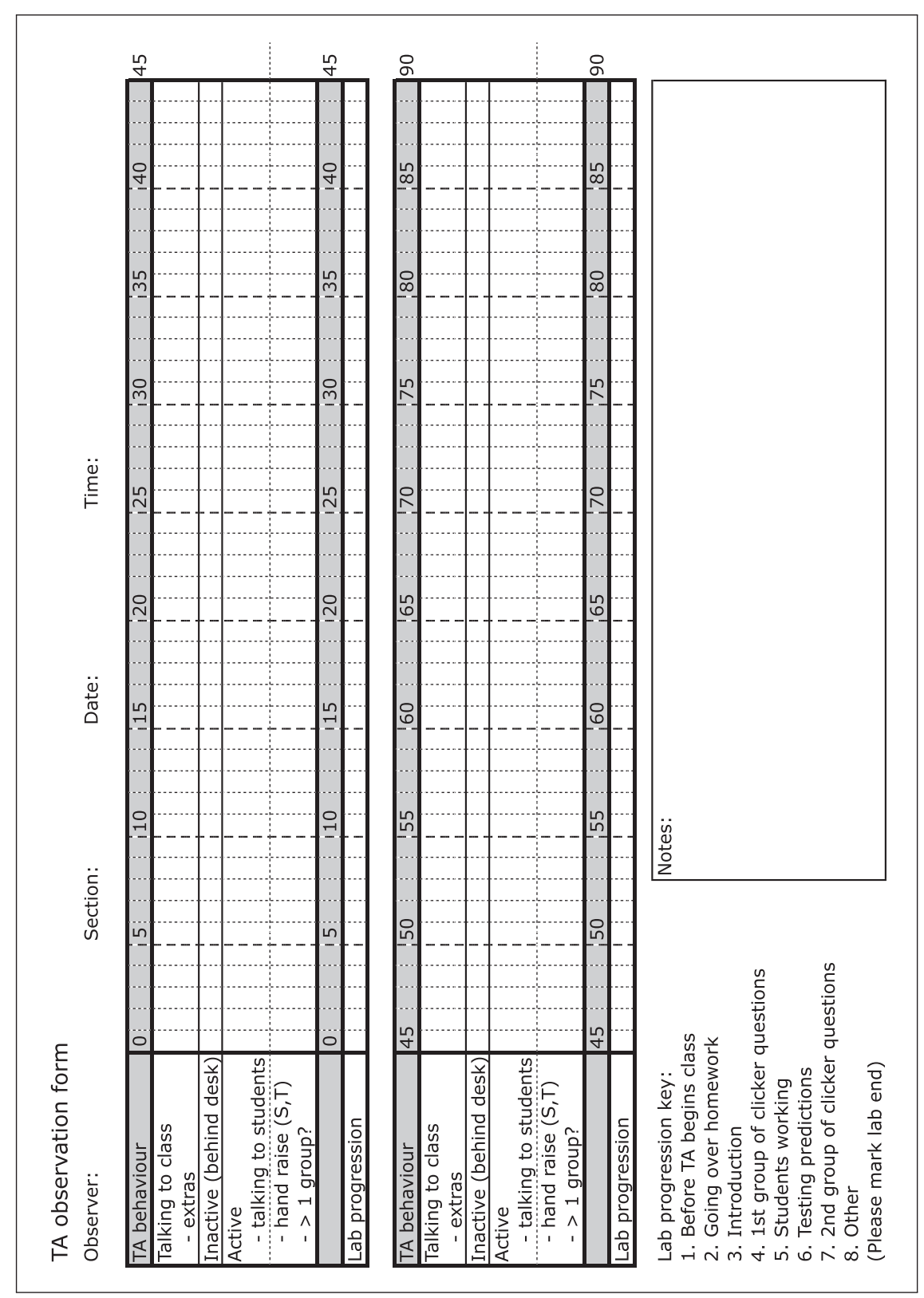

FIG. 7. The TA observation form. To complete the form, the observer shades in the areas of the timeline corresponding to the TA's actions during the lab, giving a record of the TA's behaviors during the lab. 


\section{APPENDIX C: RECORDED TA BEHAVIORS THAT WERE OUTSIDE THE SCRIPT OF THE LAB}

Here we give a list of the distinct TA behaviors that were noted as extras by the observers.

- Interrupted class to make announcement.

- Gathered class at the front chalkboard.

- Pointed out error in handout by using overhead.

- Going over method of solving clicker.

- Asked other TA question in front of everyone to spark explanation.

- Using chalkboard to explain previous week's homework.

- Asking questions to students during the explanation.

- Asking students if material is clear.

- Students discussing clicker questions with peers.

- Soliciting student responses in a class discussion.

- Using chalkboard for an explanation in an individual interaction.

- Explaining clicker question at chalkboard.

- Working on overhead to show predicted versus average.

- Other TA comments during class discussion.

- TA jumps in while other TA is leading a discussion.

- Showed video clip to motivate lab.

- Banter with TA partner during class discussion.

- TA says to class: "Today is my favorite day. I hope it's yours too."

- Impromptu discussion with the chalkboard about some student questions.

- Playing music during the lab.

- Used projector.

- Used Matlab.

- Brief Excel tutorial on computer.

- Gave students 30 seconds to individually discuss a question during a classroom discussion.

- Using in-class cameras.

- Referring to material shown on projector. 


\section{APPENDIX D: ON-OFF TASK FORM}

In this Appendix, in Fig. 8, we provide a copy of the on-off task form, used to record student engagement.

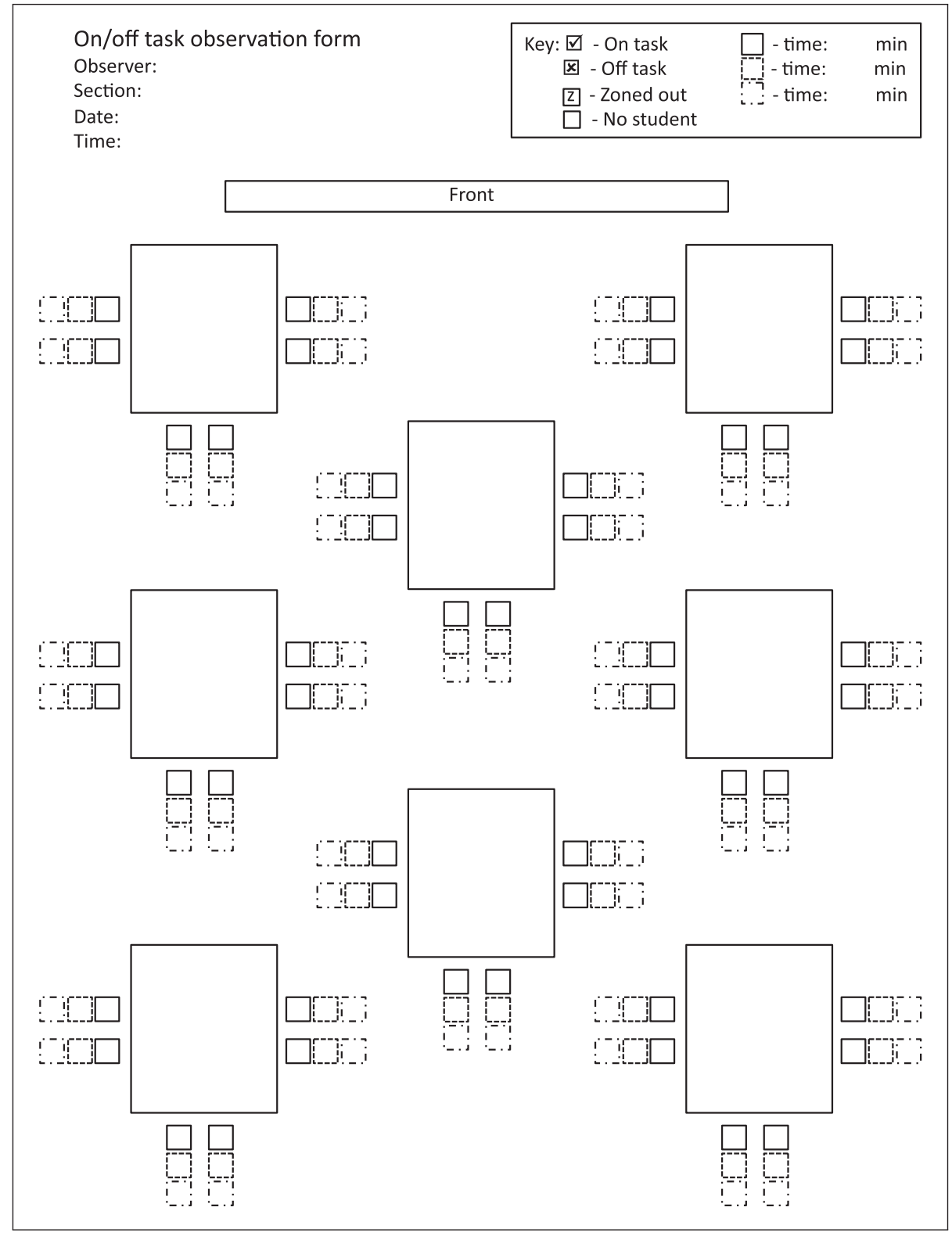

FIG. 8. The on-off task form. The form is a spatial map of the lab on which the observer records student engagement. Each form allows three snapshots of engagement to be recorded.

\section{APPENDIX E: EXAMPLE QUESTIONS FROM THE LAB EXAM}

These are three example questions from the lab skills exam given during the first and last week of the lab.

(1) Three environmentalists want to evaluate whether summers in Vancouver got warmer during the 20th century (1900-2000). They can choose one of the following data sets. Which data set should they analyze?
(a) $1980,1985,1990,1995,2000$
(b) 1906, 1907, 1908, 1909, 1999
(c) 1900,2000
(d) $1920,1940,1960,1980$ 
(2) John and Lesley measured the length of the corridor in their dorms. Each of them measured the distance three times: John measured: $10 \mathrm{~m}, 85 \mathrm{~m}, 43 \mathrm{~m}$. Lesley measured: $43 \mathrm{~m}, 45 \mathrm{~m}, 44 \mathrm{~m}$. Which of the following values is the closest to the actual length of the corridor?
(a) $43 \mathrm{~m}$, the only result that repeats more than once.
(b) $44 \mathrm{~m}$, the average of Lesley.
(c) $45 \mathrm{~m}$, the average of all values.
(d) $46 \mathrm{~m}$, the average of John.
(e) $47.5 \mathrm{~m}$, the middle between the lowest (10) and the highest (85).

(3) Dave and Jill measured the friction coefficient between two blocks of the same material. According to the textbook, the coefficient for two pieces of wood is 0.4 . They argued how many times they should measure the coefficient until they can stop measuring. Which of the following answers is most correct?

(a) After they receive the same values twice.

(b) When they notice that results converge to a single range.

(c) After two measurements.

(d) When they receive 0.4 .
[1] E. Seymour, Partners in Innovation: Teaching Assistants in College Science Courses (Rowman \& Littlefield, Lanham, Maryland, 2005).

[2] G. D. Kuh, T. M. Cruce, R. Shoup, J. Kinzie, and R. M. Gonyea, Unmasking the effects of student engagement on first-year college grades and persistence, J. Higher Educ. 79, 540 (2008).

[3] C. R. Pace, The Undergraduates: A Report of Their Activities and Progress in College in the 1980's, Tech. Rep. (Center for the Study of Evaluation, Graduate School of Education, Los Angeles, 1990).

[4] R. M. Goertzen, R. E. Scherr, and A. Elby, Accounting for tutorial teaching assistants' buy-in to reform instruction, Phys. Rev. ST Phys. Educ. Res. 5, 020109 (2009).

[5] E. R. Singer, Espoused teaching paradigms of college faculty, Res. High. Educ. 37, 659 (1996).

[6] K. Trigwell and M. Prosser, Congruence between intention and strategy in university science teachers' approaches to teaching, Higher Educ. 32, 77 (1996).

[7] B. T. Spike and N. D. Finkelstein, Examining the Beliefs and Practice of Teaching Assistants: Two Case Studies, AIP Conf. Proc. 1289, 309 (2010).

[8] B. T. Spike and N.D. Finkelstein, Toward an analytic framework of physics teaching assistants' pedagogical knowledge, AIP Conf. Proc. 1413363 (2012).

[9] R. M. Goertzen, R.E. Scherr, and A. Elby, Tutorial teaching assistants in the classroom: Similar teaching behaviors are supported by varied beliefs about teaching and learning, Phys. Rev. ST Phys. Educ. Res. 6, 010105 (2010).

[10] N. M. Speer, Issues of Methods and Theory in the Study of Mathematics Teachers' Professed and Attributed Beliefs, Educ. Studies Math. 58, 361 (2005).

[11] N. M. Speer, Connecting Beliefs and Practices: A FineGrained Analysis of a College Mathematics Teacher's Collections of Beliefs and Their Relationship to His Instructional Practices, Cognit. Instr. 26, 218 (2008).

[12] R. E. Scherr, R. S. Russ, T. J. Bing, and R. A. Hodges, Initiation of student-TA interactions in tutorials, Phys. Rev. ST Phys. Educ. Res. 2, 020108 (2006).

[13] E. A. West, C. A. Paul, D. Webb, and W. H. Potter, Variation of instructor-student interactions in an introductory interactive physics course, Phys. Rev. ST Phys. Educ. Res. 9, 010109 (2013).

[14] E. Patitsas, A case study of environmental factors influencing teaching assistant job satisfaction, in Proceedings of the Ninth Annual International Conference on International Computing Education Research, ICER '12 (ACM, New York, NY, USA, 2012), pp. 11-16.

[15] G. DeBeck and D. Demaree, Teaching assistant-student interactions in a modified SCALE-UP classroom, AIP Conf. Proc. 1413, 167 (2012).

[16] G. D. Kuh, What student affairs professionals need to know about student engagement, J. Coll. Student Dev. 50, 683 (2009).

[17] A. W. Chickering and Z.F. Gamson, Seven principles for good practice in undergraduate education, AAHE Bull. 3, 7 (1987).

[18] D. K. Duncan, A. R. Hoekstra, and B. R. Wilcox, Digital Devices, Distraction, and Student Performance: Does InClass Cell Phone Use Reduce Learning?, Astron. Educ. Rev. 11, 1 (2012).

[19] F. Sana, T. Weston, and N. J. Cepeda, Laptop multitasking hinders classroom learning for both users and nearby peers, Comput. Educ. 62, 24 (2013).

[20] R. S. Baker, A. T. Corbett, K. R. Koedinger, and A. Z. Wagner, Off-task behavior in the cognitive tutor classroom: when students game the system, in Proceedings of the SIGCHI Conference on Human Factors in Computing Systems (ACM, New York, 2004), pp. 383-390.

[21] For each lab, the TAs are provided a TA version of the lab activity, with timing notes and comments about facilitation. See Appendix A for an excerpt from the TA copy of the lab worksheet.

[22] W. B. MacLeod, D. L. Butler, and K. D. Syer, Beyond Achievement Data: Assessing Changes in Metacognition and Strategic Learning. Presented as Part of a Coordinated Symposium at the Annual Meeting of the American Educational Research Association, New York, 1996.

[23] I. Roll, V. Aleven, and K. R. Koedinger, Instruments and challenges in assessing help-seeking knowledge and behavior, edited by I. Roll and V. Aleven, in Proceedings of the 
Workshop on Metacognition and Self-Regulated Learning in Educational Technologies (SRL@ET), in conjunction with the International Conference on Intelligent Tutoring Systems (ITS), Montreal, Canada, 2008, pp. 41-50.

[24] N. G. Holmes, M. Martinuk, J. Ives, and M. Warren, Teaching Assistant Professional Development by and for TAs, Phys. Teach. 51, 218 (2013).

[25] J. Ocumpaugh, Baker-Rodrigo Observation Method Protocol (BROMP) 1.0. Training Manual version 1.0, Baker-Rodrigo Observation Method Protocol (BROMP) 1.0. Training Manual version 1.0, Tech. Rep. (EdLab., New York, NY, Ateneo Laboratory for the Learning Sciences, Manila, Philippines, 2012).

[26] J. Ocumpaugh, R. Baker, S. Gaudino, M. Labrum, and T. Dezendorf, in Artificial Intelligence in Education, Lecture Notes in Computer Science, Vol. 7926, edited by H. Lane, K. Yacef, J. Mostow, and P. Pavlik (Springer, Berlin, Heidelberg, 2013), pp. 624-627.

[27] R. S. Baker, A. T. Corbett, I. Roll, and K. R. Koedinger, Developing a generalizable detector of when students game the system, User Modeling and User-Adapted Interaction 18, 287 (2008).

[28] J. Day and D. Bonn, Development of the Concise Data Processing Assessment, Phys. Rev. ST Phys. Educ. Res. 7, 010114 (2011).

[29] A.E. Lawson, The development and validation of a classroom test of formal reasoning, J. Res. Sci. Teach. 15, 11 (1978).

[30] R. E. Scherr and H. G. Close, Transformative professional development: cultivating concern with others' thinking as the root of teacher identity, edited by K. Gomez, L. Lyons, and J. Radinsky, in Proceedings of the 9th International Conference of the Learning Sciences-Volume 1 (ICLS '10) (International Society of the Learning Sciences/ACM DL, Chicago, Illinois, 2010), pp. 388-395.

[31] The proportion of TA-initiated interactions we recorded agrees with the proportion reported in Ref. [12].

[32] M. T. H. Chi, Active-constructive-interactive: A conceptual framework for differentiating learning activities, Topics Cognit. Sci. 1, 73 (2009). 